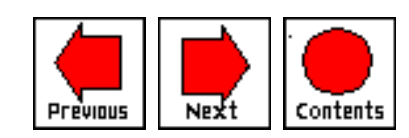

\title{
COMPUTER INTERACTION IN THE USE OF RADIOMETRIC CALIBRATION FOR THE CASSINI SPACE FLIGHT PROBE
}

\author{
Kenneth A. Brown $\{\{$ bio $\}\}$ \\ Clark Atlanta University \\ Atlanta, GA \\ Editor: Max M. North
}

\begin{abstract}
In the area of Radiometric Calibration for Space Flight Hardware, there is a need for accurate data manipulation and analysis. At the Jet Propulsion Laboratory in Pasadena, CA, there is an advanced system of calibration and analysis of spacecraft data designed by the Planetary Instruments Group of the Space Instruments Implementation. We examine a problem with the user interface. As data is downloaded in this system, it must be physically examined for "good" and "bad" data points. This paper describes how to make this process more efficient. With the use of a light pen or touch-screen CRT the choice of data points can be quickly and accurately chosen or discarded. The article provides a description of the process of data acquisition as well as contributions toward the design of a more "friendly" data calibration procedure.
\end{abstract}

\section{Introduction}

There has been much work on how to use a light pen or touch-screen CRT for a more productive work environment. Most applications have focused on nonscientific based projects, including making word processors more "user friendly" and manipulating spreadsheet operations. This paper describes a more useful way of confronting scientific data.

The National Aeronautics and Space Administration (NASA) and the Jet 
Propulsion Laboratory (JPL) in Pasadena, CA, are in the midst of preparing a spacecraft for scientific exploration of the Saturian system. On this spacecraft are eleven (11) large instruments. One of them is the Visible and Infrared Mapping Spectrometer (VIMS). The VIMS has a 2-axis scan mechanism which "maps" out the surface of a given body or satellite (Figure 1). Before this system can launch, however, numerous tests need to be performed. I shall discuss the procedure for one of these tests, and how we propose to make enhancement in the user interface during these tests.

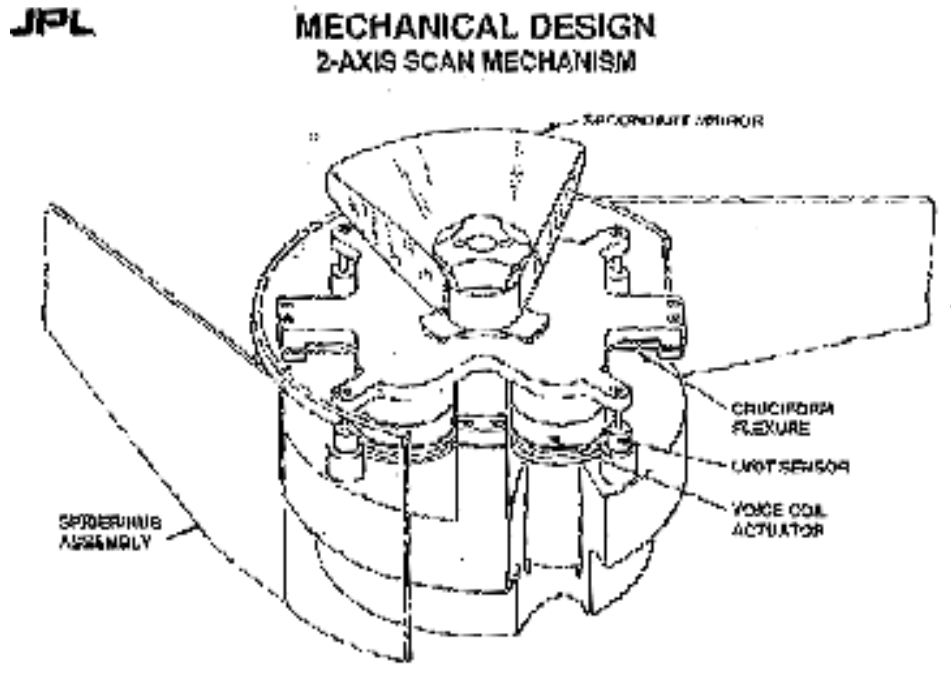

Figure 1: Cassini VIMS 2-axis scan mechanism.

The equipment being used for this calibration includes the VIMS 2-axis scanning mechanism, an Apple Quadra 800 computer, and customized data acquisition software written in LabVIEW programming language. All data acquisition and calibration tests are performed at pressure in a thermal vacuum chamber located at JPL.

\section{Previous Work}

Why are we even worried about redesigning a system to pick out points on a computer screen? The simple answer: to make the work more productive. Allowing the user to be comfortable both physically and mentally will result in "maximum functionality at minimal cost." This is the goal of the system modification [Haubner]. In Computers At Work [Osborne] the author describes the advantages of using a touch-screen CRT or light pen. The advantages of these types of input devices are (1) they are easy to use, (2) they are fast to use, (3) they minimize errors, (4) they are flexible, and (5) the screens are favored by system users. They give the user one more tool in an array to help solve his problem. The user can not only use his eyes to identify a problem, he can use his eyes to help solve problems as well [Jacob]. It is noted that these devices do 
have a drawback: there is a slightly higher cost for hardware and an increased programming time. Nevertheless, the system is still relatively easy to implement and has a low long- term cost [Craine].

\section{Our Approach}

The primary test needed for this instrument is a radiometric calibration. The test produces the radiometric calibration coefficient which converts detector output (DN) to radiance for each VIMS pixel. In short, this test checks and corrects the accuracy of the scanning mechanism for radiometric measurements.

First a beam of light is focused towards the instrument. Then the mechanism "steps" through known distances. The corresponding distances are converted into pixel coordinates and downloaded. The data is then analyzed for the validity of each measurement.

It is at this point a new user interface is implemented. After the data points are plotted on the screen (figure 2), the user examines them. Here the user chooses points deemed as "good" data points and "bad" data points. The "good" points are kept, while the "bad" points are deleted from the screen and the data file. In the past the user had to first identify the points and then go into the spreadsheet to search for the points that needed to be deleted. The user then opened up the data file and searched for the correct coordinates. After deleting the "bad" points from the data file, the user then had to re-format the spreadsheet and plot the new, adjusted points. Following this, the user would again look to see if there were any points missed. This process was repeated until all the "bad" points were deleted.

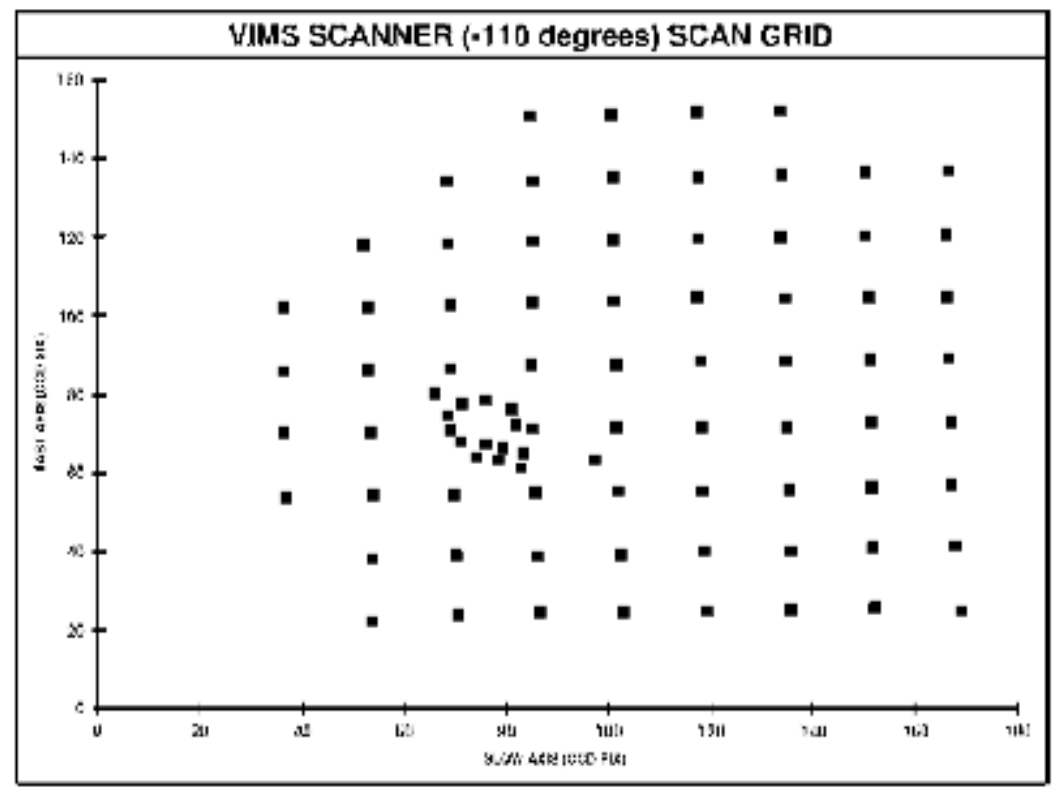


Figure 2: Points of raw data.

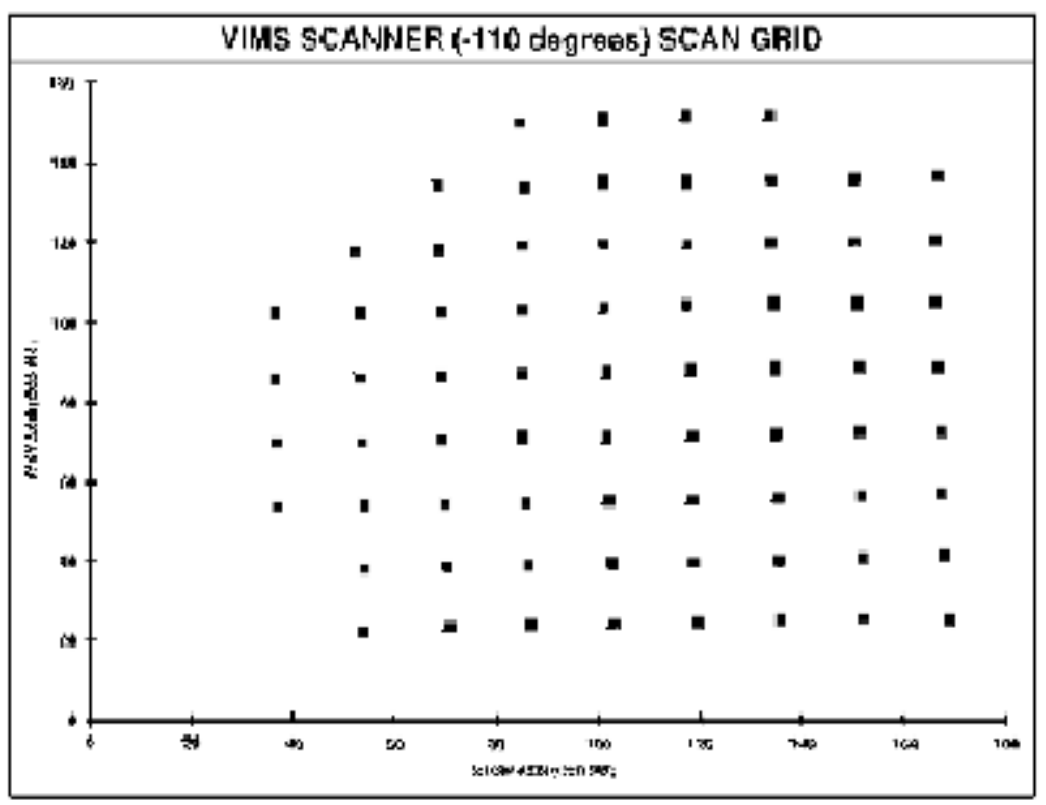

Figure 3: Bad data points removed from the raw data.

It is our goal to introduce an updated or modified userinterface to make this task simpler and more efficient. Instead of taking the time to calculate which point is good and which point is bad, we implement a new userinterface system of a touch-screen CRT or light pen. These systems give the user instantaneous control over his working environment. The plotted data grid is first observed for any apparent problems. The user then chooses the points he wants to keep. After tapping these points with the either a light pen or finger on the screen, the points are quickly and efficiently deleted from the plotted grid and updated in real-time.

If somehow the wrong point is chosen, the user still has the opportunity to recall or "undo" his actions with a simple keystroke. After the problem points are eliminated and the grid updated, the information is ready to be transferred to the next step of the calibration procedure (figure 3). Because the data file is updated in the plotted screen data as well, the user no longer has to go into the "raw" file to manually remove bad data coordinates. This saves time and again increases productivity.

\section{Preliminary Results}

Although this system has not been fully integrated into the existing calibration unit, there has been communication with the scientists and engineers who use it. The users were asked for their input and opinion on how these changes might affect productivity and accuracy of the calibrating procedure. Of the six 
persons surveyed, all thought that implementing the changes described above would greatly increase the speed and accuracy of the data analysis. Since this system will be used for more than just the radiometric calibration of the Visual and Infrared Mapping Spectrometer, upgrades and improvements are always key issues of discussion. The issue of how to make a transition from the system already in place poses a most serious question. Currently serious thought is being given to the idea of updating or changing the user interface for better computer interaction.

\section{ACKNOWLEDGMENTS}

This research is being conducted at the Jet Propulsion Laboratory in Pasadena, CA for the National Aeronautics and Space Administration under the management of David Jeurgens in the Space Instruments Implementation Section. The research is also supported in part by the National Physical Science Consortium and the Center for Theoretical Studies of Physical Systems. Thanks to Dr. David Thomas for his support and guidance in the Optical Science Section at JPL, and to Dr. Max North in the Computer Science Department at Clark Atlanta University, Atlanta, GA.

\section{REFERENCES}

Craine, J. Ffynlo, Microcomputer in Engineering and Science, Addison- Wesley, 1985.

Haubner, Peter, Design Of Dialogues For Human-Computer Interaction, Galer, Margaret, ed. 1992.

Methods and Tools in User-Centred Design for Information Technology, Elsevier Science Publishers B.V.

Jacob, Robert J.K., "Integrality and Separability of Input Devices", $A C M$ Transactions on Computer-Human Interaction 1 (March): p.3-26, 1994. Osborne, David J., Computers at Work: A Behavioral Approach, John Wiley \& Sons, 1985.

Pinson, James L., Designing Screen Interfaces in C, Yourdon Press, 1991.

\section{BIOGRAPHY}

Kenneth A. Brown holds an MS degree from Clark Atlanta University, Atlanta, GA, in Applied Physics and a BS degree in Physics and Computer Science from Morehouse College, also at Atlanta, GA. He expects to complete his PhD in the 
Department of Atmospheric Science at the University of California - Los Angeles in the year 2000. He has worked on various projects at the Jet Propulsion Laboratory, Pasadena, CA, each summer since 1988, and as an undergraduate worked for Hughes Aircraft, Longbeach, CA.

Contact information:

The National Physical Science Consortium

The Center For Theoretical Studies of Physical Systems Physics Department Clark Atlanta University

J.P. Brawley @ Fair Street

Atlanta, GA 30314

Email: Ken.Brown@Jpl.Nasa.Gov

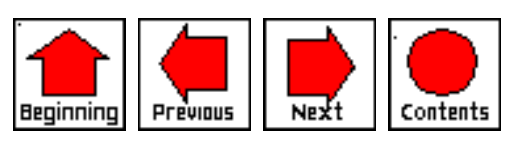

